The first known GPCR structure, published in low resolution in 1993 (ref. 4) and in high resolution in 2000 (ref. 5), was of the bovine version of rhodopsin, the photoreceptor that enables vision in low light conditions. Rhodopsin, however, is an unusual GPCR because it is particularly stable and is expressed in high enough concentrations to be collected from natural sources - two characteristics not usually associated with this protein family. As such, "rhodopsin didn't tell us how to get structures for other GPCRs", says Kobilka. It wasn't until 2007 that researchers solved the structure of a second $\mathrm{GPCR}^{6}-$ the human $\beta_{2}$-adrenergic receptor, which is involved in cardiovascular and pulmonary function. Kobilka led a team that designed an antibody to bind two of the protein's helices, stabilizing the receptor and providing a polar surface that helped crystals to form ${ }^{7}$. In other work, Kobilka and the Scripps Research Institute's Stevens solved a high-resolution structure from another crystal, in which the protein was stabilized by replacing an intracellular loop with another protein ${ }^{6,8}$.

Researchers are also hunting for compounds that can boost proteins' stability without adding another protein, a strategy that has allowed Stevens to obtain a portrait of another important GPCR, the $\mathrm{A}_{2 \mathrm{~A}}$ adenosine receptor ${ }^{9}$, which is involved in many physiological processes and is blocked by caffeine. Receptos, a drug development company co-founded by Stevens and based in San Diego, California, used the same method to solve the structure for sphingosine1-phosphate receptor subtype 1 , a drug target for multiple sclerosis. This year, the company announced a clinical drug candidate designed with reference to this structure.

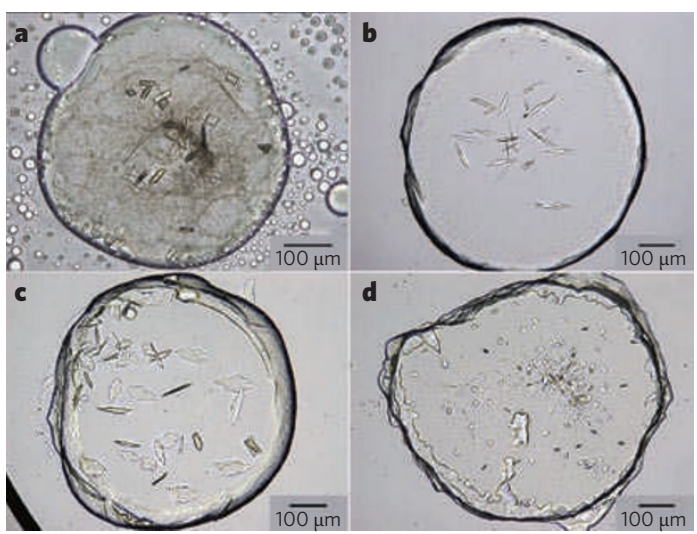

$a$, adrenoreceptor $\beta_{2} A R ; b$, adenosine receptor $A_{2 A} A R$; $c$, chemokine receptor CXCR4; $d$, dopamine receptor $D_{3}$.

Another tactic is to stabilize GPCRs through targeted mutagenesis rather than through third-party agents such as antibodies and extra proteins. Researchers led by Chris Tate and Gebhard Schertler at the Medical Research Council's Laboratory of Molecular Biology in Cambridge, UK, for example, identified a handful of mutations in GPCRs that boost stability with no apparent effect on function ${ }^{10}$. In 2007, Tate co-founded Heptares Therapeutics in Welwyn Garden City, UK, which uses the stabilized GPCRs, known as StaRs, to inform drug design and has solved several crystal structures of GPCRs with bound ligands.

\section{Sticking with a membrane}

Techniques for purifying membrane proteins without denaturing them go beyond tool compounds and engineering. Anatrace in Maumee, Ohio, part of Affymetrix, based in Santa Clara, California, sells detergents and lipids used for solubilizing and stabilizing proteins, including
Chobimalt, a water-soluble cholesterol derivative; A8-35, a polymer that wraps itself around the membrane protein; and tripod amphipiles, which limit protein mobility and interactions. In 2007, the company launched three or four new products for membrane proteins; last year, it rolled out twenty. The growing number of publications and tools has brought in scientists who previously restricted themselves to soluble proteins, says Ben Travis, the company's research and development manager. "They're finding the membraneprotein field more accessible," he says.

Perhaps the biggest shift in the field is the ability to accommodate membrane proteins' structural need for fat. "Before, people tried to purify membrane proteins so they didn't have lipids associated with them, but now we know that it probably isn't a good idea," says Stephen White, a biophysicist at the University of California, Irvine.

To address this, many structural biologists have turned to a technique called lipidic cubic phase (LCP) crystallization, also known as in meso crystallization. In this technique, proteins are dissolved in lipids to form membrane-like bilayers around water-filled cavities. This mixture feeds lipids and proteins into crystals as they grow. The idea that one could obtain crystals from a protein embedded in a bilayer was "pretty radical", says Bowie, who has developed a variant of the technique using bicelles of lipid and detergent. "A number of protein structures would remain unsolved without the LCP method."

The LCP mixture, however, is incredibly difficult to work with. "You end up with something that looks and feels like very sticky toothpaste," says Martin Caffrey, a biochemist from Trinity

\title{
CRYSTAL-CLEAR IMAGES
}

Getting proteins to form crystals is only one step for the structural biologist. The next step to sleuthing out a protein's structure involves placing the crystals in an intense beam of $X$-rays. This radiation bears little resemblance to the broad, diffuse X-rays used in medicine: the powerful X-rays that work best for protein crystallography are produced at giant facilities called synchrotrons, of which only a few dozen exist.

At the Advanced Photon Source synchrotron at Argonne National Laboratory, Illinois, for example, electrons race around a 1.1-kilometre track at close to the speed of light. Radiation generated from the electrons is collected into a 70-metre beamline, which focuses X-rays into a 25-squaremicrometre area where crystals can be positioned for analysis. Proteins in the crystal scatter the $\mathrm{X}$-rays as they pass through, and researchers can decipher a protein's structure from the resulting diffraction pattern. To generate a complete pattern, the crystal must be rotated within the beam so that X-rays pass through in different orientations. The process requires precision: researchers have to collect enough data to solve a structure, while limiting radiation

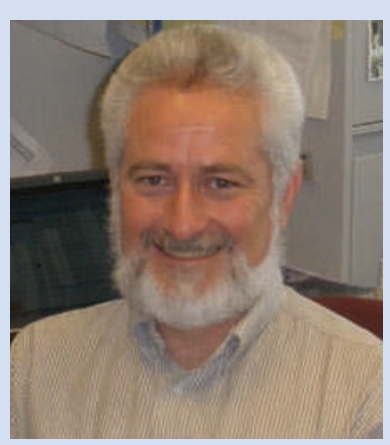

Robert Fischetti matches crystals with X-rays. damage to the crystal.

Technologies for manipulating crystals and keeping them at temperatures below $0^{\circ} \mathrm{C}$ to decrease radiation damage have got better, but the most dramatic improvement is that experiments can now be done using very small crystals or crystals with many poorly diffracting regions, says So Iwata, who heads the Human Receptor Crystallography Project at the Japan Science and Technology Agency in Kyoto. "Crystals that would have been turned away ten years ago are welcome now," he says.

Still, a crystal must be as wide as or wider than the beam passing through it to generate a reliable diffraction pattern. That's a problem, because crystals of membrane proteins tend to be small, says Robert Fischetti, a senior scientist at Argonne National Laboratory, which has produced data for crystal structures of several membrane proteins, including the $\beta_{2}$-adrenergic receptor (S. G. Rasmussen et al. Nature 450, 383-387; 2007). Technologies such as lipidic cubic phase crystallization have helped researchers to grow crystals, he says, but these are often only 5-10 micrometres across, a tenth the size of most crystals submitted for analysis and much smaller 